\title{
A critical look at risk analysis of disasters
}

\author{
A. Lepikhin \\ SKTB "Nayka", Krasnoyarsk Science Center, Russia
}

\begin{abstract}
Recent natural and man-made worldwide disasters show an increased vulnerability of society. The risk analysis of disasters is an important component of life quality for all industrial societies. However, comparison between hazards, vulnerabilities and risks causes many difficulties. A critical look at the methodologies for risk assessment of disasters is presented in this paper. The main issues of safety measures and risk-analysis are considered. Special attention is paid to survivability and robustness analysis of infrastructure, population and environment at a disaster occurrence.
\end{abstract}

Keywords: hazards, disasters, risk analysis, damages, critical infrastructure, critical technologies.

\section{Introduction}

Natural and man-made disasters are permanent hazards for life on the Earth. The disasters can cause failure of engineering structures, disruption of technological processes, damage of life support systems, deaths of people and environment degradations. The traditional concept of the disaster analysis is based on an assessment of risk as the probability of a damage or loss. A comparative risk analysis is an essential part of a security policy in many countries. The purpose of risk analysis is the disaster reduction for sustainable development.

The traditional risk analysis is based on integration of predictive (probable) and retrospective (statistical) estimation methods of rare-to-happen but high consequence events. The core of risk analysis includes three parts: hazard identification, risk analysis and risk evaluation. The choice of a risk analysis method depends on features of a system to be analyzed and the purposes of study. The natural hazards considered are droughts, fires, floods, earthquakes, heat waves, hurricanes, typhoons and cyclones, tornadoes, winter storms, volcanoes. The man-made hazards are fractures or damages of nuclear plants, 
power generation and distribution facilities, chemical plants, manufacturing facilities, high-tech facilities, offshore platforms, pipelines, dams, buildings.

Currently intensive researches and engineering investigations are being carried out on probabilistic quantification of natural and man-made hazards and analysis of a broad range physical base of disaster reduction.

The saturation of technical sphere with dangerous objects is accompanied by a steady increase in number and severity of the man-made disasters. Continual complication of technical systems and increased safety requirements come into collision with technical and scientific resources to provide these requirements. Despite of outstanding achievements in scientific and technological research, the disaster statistics in industrialized countries displays that opportunity to parry hazards turned out to be limited. The traditional safety measures approaches based on elimination of disaster causes basically ran dry. To resolve the deadlock new risk-analysis paradigm and theoretical approaches to safety measures and stable development have to be elaborated.

The aim of this study is a critical look at theoretical conceptions of risk and risk analysis of disasters.

\section{Brief description of theoretical background}

Theoretical investigations of the disaster risk analysis include several aspects. One of them is the Mathematical Theory of Reliability (Barlow and Proshan [1]), which became the basis for the development of a number of statistical risk evaluation methods. The Theory of Normal Accidents (Perrow [2]) represents a development of this field. According to the theory, a complicated dynamics of states of multi element systems is the dominant factor for an accident formation. The analysis of such dynamics requires enumeration of a variety of the initial states and prediction of their further trajectories. This is possible for wellexamined objects only. Risk analysis using the event-tree and fault-tree (ET/FT technology) is one of well-known practical realizations of this theory.

The Theory of Self-organized criticality (Bak et al. [3]) gives another approach to risk analysis. The main point of the theory is the fact that "small causes" in large systems can have "big effects" due to an avalanche of branching cause-and-effect relations. This theory explains well a high sensitivity of systems to fluctuation of determinative parameters. The theory allows studying the vulnerability of self-organized systems and occurrence of a disaster near the critical point. However, a practical implementation of the theory is not realized yet.

At the end of the XX century a new approached was developed - the Mathematical Theory of the Catastrophes (Arnold [4], Poston and Stewart [5]). This theory qualitatively describes the state dynamics of complex systems, determines the boundaries of steady states and proximity to catastrophe events. The disadvantage of the theory is that to predict the catastrophe one has to formulate analytical functions of system states, whose parameters are difficult to substantiate. 
Recently, one more mathematical theory oriented to risk-analysis is actively forming - the Theory of the Dynamic Systems (Malinetskiy and Potapov [6]). According to this theory, two specific types of the state dynamics are assigned for complex systems. The first type is a smooth change of conditions when the system can be approximately described as being determinate and methods of the dynamic systems theory are applicable for its characterization. The second type the bifurcational events - due to which the system can, with some probability, be in different conditions. The choice of these conditions is not known in advance. This type of system dynamics doesn't still have an analytical description, which is suitable for the practical implementation.

The theories above can be used for the risk-analysis of both, natural and manmade disasters.

Limitations of these theories predetermined the formation of phenomenological and semi-empirical risk theories. Theoretical basis of this approach was worked out by Bolotin [7] and Makhutov [8]. The approach assumes that a technical system disaster is a consequence of damage accumulations and process deviations, which define the state dynamics of the system. Significant practical results were obtained using this theory (Lepikhin et al. [9]).

The analysis of the mentioned theories shows that the problems of disaster risk evaluation have mainly been formulated and new approaches to their solution are being developed. At the same time, awareness of impossibility in principle to eliminate disasters leads to formulation of new scientific tasks. These are the vulnerability analysis, survivability and robustness of objects, critical infrastructure and environment under impact of natural and man-made hazards. Vulnerability analysis problems are being actively studied in the USA (Center for Risk Research, Engineering Risk Research Group) and the EU (Institute of Hazard, Risk and Resilience). In Russia the system survivability and robustness are generally studied at universities and academic laboratories. There is no common conceptual approach to the disaster risk analysis. The methodology of the vulnerability analysis and theory of survivability are at the initial stage when the basic concepts and their application fields are being defined. There is a lack of vulnerability and robustness criteria, and survivability models based on the fundamental representations of nature and mechanism of the disasters. This impedes realization of the basic principles of security policy such as a high reliability at a given life-time (safe-life) and a high survivability with existence of damages (fail-safe). This in turn deters the creation of new objects and systems ensuring the development of new technologies (high-speed transport, thermonuclear power etc.). As a rule, such objects are critically and strategically important technical systems. Accidents on the critically important technical systems can lead to the loss of control on regional or national economics, negative changes in the safety level for a long-term period. Examples are Three Mail Island and Chernobyl nuclear power plant disasters, the disaster on the Sayano-Shushenskaya hydropower plant.

The theory and methods of risk analysis are particular important due to rapid development of the critical technologies: new materials design, information and 
communications, biotechnology and sciences, aeronautics and surface transportation, energy and environment. As opposite to technical systems the critical technologies can influence all the spheres of people life and can generate the global hazards that can't be parried without the priority development of safety and risk theories.

\section{Hierarchical multiobjective analysis of large-scale systems}

Three basic questions should be taken into consideration while elaborating mathematical risk-analysis models:

1. What do "risk" and "uncertainty" mean?

2. What are purposes and objectives of the risk-analysis?

3. What does the model express in the risk-analysis context?

The answer to the first question is the following. Modern concept of the technogenic safety is based on the following principles. Firstly, a disaster of any system is possible. Deterministic or random uncertainties of different nature (defects, damages, deviation of working parameters, human errors etc.), which originate during design, manufacturing or operation activities, are the main sources of disasters. Secondly, the risk defined in a form of probability of damage or loss is a quantitative measure of the disaster possibility. Thirdly, it is assumed that the acceptable risk level can be set.

Uncertainties play the role of the perturbing factors leading to undesirable consequences. If the undesirable consequences are measured as $C$ and the probability as $P$, the risk $R$ on $N$ set of possible disaster "trajectories" can be presented in a classical way:

$$
R=\sum_{i=1}^{N} C_{i} P_{i}
$$

Risk-analysis of disasters consists in the solution of the task (1) for every given type of hazards. The task can be fulfilled in a form of Probabilistic Risk Assessment (PRA) or Probabilistic Safety Assessment (PSA).

The answer to the second question is the following. Risk-analysis in a modern definition is a part of a decision making system at the disaster occurrence and a tool for the hazard assessment. At the first case, the risk allows to choose optimal protection methods against the disaster or to prevent it. At the second case, risk is minimized on the limited resources.

The answer to the third question seems to be more complicated. Contemporary hazards are associated with accidents on large scale systems (LSS). Decomposition tasks of such structures appear to be multivariant and result in ambiguous solutions. Due to complexity and statistical nonreproductivity of the LSS state dynamics, element variety and disaster mechanism multiplicity, development of universal risk models seems to be hardly probable. More promising is the creation of specific risk models based on representation of the LSS as a structure $\Sigma$, which consists of subsystems $\sigma$ and elements $e$ : 


$$
\Sigma=\bigcup_{i} \sigma_{i}\left(\bigcup_{j} e_{i j}\right), \quad i=1, n, \quad j=1, m .
$$

These models should represent the decomposition of R-characteristics of the structure (2) in a form of:

$$
R_{\Sigma} \rightarrow\left\{R_{i}\right\} \rightarrow\left\{R_{i j}\right\} \rightarrow\left\{R_{i j k}\right\}
$$

where $R_{\Sigma}$ is an integral (system) risk, $R_{i}$ - complex (subsystem) risk, $R_{i j}$-element risk and $R_{i j k}$ - criterion risk.

The final term in (3) is the criterion risk that allows one to relate the system risk to mechanisms of disasters. We call this method as Hierarchical Multiobjective Analysis of Large-Scale Systems (HMALSS). The following features of the LSS as the objects of risk analysis should be considered while modeling the HMALSS.

Firstly, in most cases we have to analyze the situations that have never happened, for the coincidence of all the disaster conditions is basically impossible.

Secondly, the analysis is performed under conditions of high uncertainty due to the random nature of external actions and processes, multiplicity of safety criteria and objectives, as well as alternative decisions and their consequences.

Thirdly, the analysis is strictly limited in time. During the project design analysis stage theses limits are determined by the design time, during the operation stage - by the response time to react to an accident or emergency situation.

These features put in specific requirements to models, computing equipment and data base of risk analysis.

Development of the models and computational tools is related to a number of specific tasks.

The first task is to describe the LSS in terms of integrity and hierarchy. Building a computational model inclusive a big number of meaningful parameters is considered a hard problem even using modern mathematical and computer technologies. Decomposition of a system onto constituent elements is the basis of the model creation. In this context, two levels of decomposition are possible. At the first level the system is divided into blocks according to a set of variables, which define the criteria functions.

The second task consists in a formulation of risk-analysis dataware. It includes two aspects. One is related to a data processing task. The other is associated with the necessity to formulate hypothesis about states of elements, which are based on the available information. The validity of such hypothesis depends on the accuracy and completeness of the information.

The third task is related to a choice of risk criteria. It can be solved based on analysis of known indexes or developing new ones that must indicate necessary features of the limit states or limit process parameters. The choice can be nonunique or multi-objective.

Finally, the fourth task is to create a risk-analysis tool for given varying parameters. Such tool can be considered as a set of mathematical models that 
reflect the disaster mechanisms in a certain sequence of the risk-analysis process. The fact that a disaster event itself is accidental should be taken into account. The final goal is the development and realization of mathematical tools and methods that are practically applicable for a formalized risk-analysis of the LSS.

Man-made hazards have different mechanisms of occurrence. That is why analysis of the hazards is based on special classification of risk probabilistic models: trends model, extremum model, large-scale disaster model (Fig. 1).

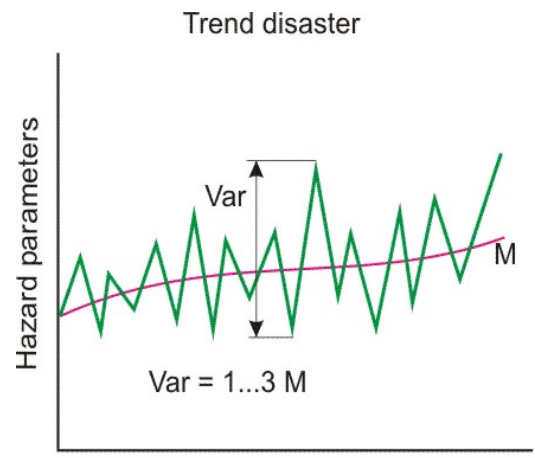

Time

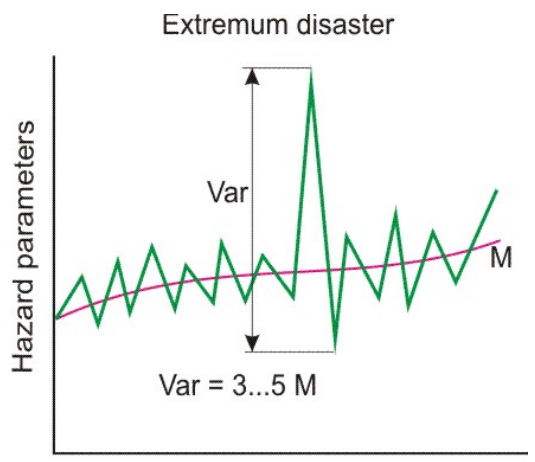

Time

Large-scale disaster

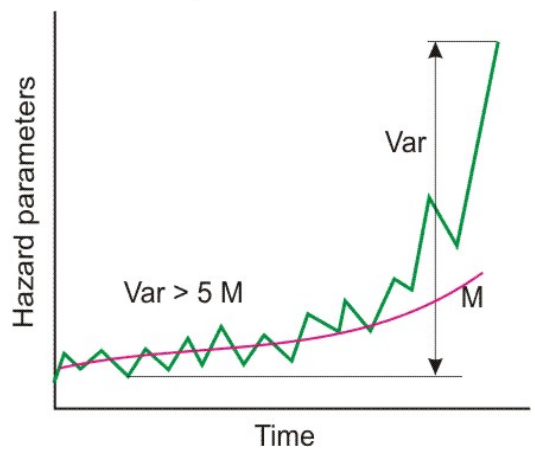

Figure 1: $\quad$ Models of disasters.

Trend model is linked to evolutional changes of system parameters. The disaster occurs when the parameters reach their critical levels. For this case, the risk assessment can be done using conventional models.

$$
R(S, t)=\prod_{k}\left[1-\Phi\left\{\frac{z_{k}-v_{k} t}{\sqrt{S_{z}+S_{v}}}\right\}\right] \sum_{k} U_{k}(S)
$$

where $v$ - speed of trends; $\mathrm{z}$ - critically parameter; $\sigma_{v}, \sigma_{\gamma}-$ dispersion of $v$ and z; $t$-time; $\Phi$ - Gauss function; $S$-impact area; $U_{k}-$ losses.

Extremum disaster is associated with occurrence of rare, extreme values of parameters. For such events, the risk assessment can be done using the Poisson's model.

$$
R(S, t)=\left[1-\exp \left\{-\sum_{k} \frac{S_{k}}{S} \int_{t} \mu_{k}(S, t) d t\right\}\right] \sum_{k} u_{k} \frac{s_{k}}{S}
$$


where $S$ - impact area; $S_{k}$ - area for which the rate intensity parameter $\mu$ was estimated; $u_{k}-$ losses.

Large-scale disaster is defined by dynamic buckling collapse of a system. There are no models developed so far for such cases.

\section{Risk assessment of disasters in regions of Siberia}

The multilevel conception represented above was used for the risk assessment of disasters on the territory of Siberia. Siberia is the largest region of Russia. Its territory is 5,1 million square kilometers. Population is 20,1 million people. GDP accounts for 11,4\% GDP of Russia. Resources make up 45-80\% of Russia's natural resources. Siberia includes such territories as: Altai Kray, Altai Republic, Buryat Republic, Chita Oblast, Irkutsk Oblast, Republic of Khakassia, Kemerovo Oblast, Krasnoyarsk Kray, Novosibirsk Oblast, Omsk Oblast, Tomsk Oblast, Tuva Republic. Natural hazards are defined by geographical and climatic conditions of the region. Man-made hazards are defined by peculiarities of economic infrastructure and distribution of industrial facilities. There are: 8 largest hydropower stations; 54 large thermal stations; more than 1200 big chemical and petrochemical objects. Most of them are located in or near metropolises. Around $60 \%$ of population lives in possible impact zones. Main natural hazards include flooding, earthquake, windstorm, wildfire, hard frost (low temperature). Amongst them flooding and wildfire are the most intensive.

Characteristic of the main man-made disasters is shown in the table 1 . The risk of natural disasters was estimated by statistical methods. The risk of manmade disaster was estimated using the HMALSS. Two disaster scenarios were examined: trend disaster and extremum disaster. Results of the risk analysis are presented in the table 2 .

Table 1: $\quad$ Man-made hazards of large-scale systems.

\begin{tabular}{|c|c|c|c|}
\hline Type of LSS & $\begin{array}{c}\text { Number of } \\
\text { objects }\end{array}$ & $\begin{array}{c}\text { Degradation, } \\
\%\end{array}$ & $\begin{array}{c}\text { Potential risk, } \\
\text { loss or life, } \\
\text { humans }\end{array}$ \\
\hline $\begin{array}{c}\text { Radiation hazardous } \\
\text { objects }\end{array}$ & 3 & 60 & 240000 \\
\hline $\begin{array}{c}\text { Chemistry hazardous } \\
\text { objects }\end{array}$ & 36 & 55 & 290000 \\
\hline $\begin{array}{c}\text { Explosion hazardous } \\
\text { objects }\end{array}$ & 56 & $55-60$ & 150000 \\
\hline Fare hazardous objects & 213 & 65 & 1794000 \\
\hline Hydropower, dams & & & \\
\hline
\end{tabular}

Generalized risk estimates were made for three hazard classes: low, medium and heavy. The results are presented in the table 3. According to the data, economic losses incurred by these hazards can reach hundreds millions or even billions of dollars and mitigation of consequences can take several years. 
Generalized risk estimates were made for three hazard classes: low, medium and heavy. The results are presented in the table 3. According to the data, economic losses incurred by these hazards can reach hundreds millions or even billions of dollars and mitigation of consequences can take several years.

Table 2: $\quad$ LSS risk assessment.

\begin{tabular}{|c|c|c|c|}
\hline Type of LSS & $\begin{array}{c}\text { Type of } \\
\text { Disasters }\end{array}$ & $\begin{array}{c}\text { Failure rate } \lambda, \\
\text { year-1 }\end{array}$ & Loss, mil $\$$ \\
\hline $\begin{array}{c}\text { Radiation } \\
\text { hazardous objects }\end{array}$ & Trend & $10-4 \ldots 10-5$ & $10-1 \ldots 101$ \\
\hline $\begin{array}{c}\text { Chemistry } \\
\text { hazardous objects }\end{array}$ & Extremum & $10-5 \ldots 10-8$ & $102 \ldots 105$ \\
\hline & Extrend & $10-3 \ldots 10-4$ & $10-3 \ldots 10-1$ \\
\hline $\begin{array}{c}\text { Explosion and } \\
\text { fire hazardous } \\
\text { objects }\end{array}$ & Trend & $10-5 \ldots 10-6$ & $101 \ldots 102$ \\
\hline & Extremum & $10-5 \ldots 10-6$ & $10-2 \ldots 101$ \\
\hline $\begin{array}{c}\text { Hydropower } \\
\text { plants, dams }\end{array}$ & Trend & $10-4 \ldots 10-5$ & $10-1 \ldots 101$ \\
\hline \multicolumn{2}{c}{ Extremum } & $10-5 \ldots 10-7$ & $102 \ldots 104$ \\
\hline
\end{tabular}

Table 3: Generalized risk estimates for risk of disasters in regions of Siberia.

\begin{tabular}{|c|c|c|c|c|}
\hline $\begin{array}{c}\text { Hazard } \\
\text { classes }\end{array}$ & Consequences & $\begin{array}{c}\text { Probability, } \\
\text { year }^{-1}\end{array}$ & $\begin{array}{c}\text { Loss, } \\
\text { mln. } \$\end{array}$ & $\begin{array}{c}\text { Recovery } \\
\text { time, } \\
\text { year }\end{array}$ \\
\hline Low & $\begin{array}{c}\text { Work disturbance } \\
\text { of an object }\end{array}$ & $10^{1} \ldots 10^{-2}$ & $10^{-1} \ldots 10^{1}$ & $<0.01$ \\
\hline Medium & $\begin{array}{c}\text { Damage of objects } \\
\text { and infrastructure }\end{array}$ & $10^{-2} \ldots 10^{-5}$ & $10^{1} \ldots 10^{2}$ & $<1$ \\
\hline Heavy & $\begin{array}{c}\text { Damage of facilities } \\
\text { and settlements, } \\
\text { impact of natural } \\
\text { environment }\end{array}$ & $10^{-5} \ldots 10^{-8}$ & $10^{2} \ldots 10^{4}$ & $\leq 5$ \\
\hline
\end{tabular}

The analysis revealed the following issues. The trend disaster model can be used in the risk analysis of the LSS, for which variation in parameters does not exceed three mean-square deviations. The extreme model can be used for the LSS when the parameter variations are within a range from three to five sigma. Finally, the dynamic LSS-model should be applied when the parameter variations exceed five sigma.

Analysis of statistics shows that the annually average number of man-made hazards and natural hazards in Siberia can amount to around 350. Total economic losses from these events exceed 3.3 billion dollars. The individual risk 
of death from industrial accidents equals to $1.65 \times 10^{-5}$ year $^{-1}$. The societal risk is about $2.3 \times 10^{-2}$ per. $\times$ year $^{-1}$. The risk of living conditions temporal disruption due to failure of life support systems is also quite high.

\section{Conclusion}

In conclusion, the following should be mentioned. Mathematical modeling is the most effective tool for a modern risk analysis of the LSS. The development of this direction is connected with progress in deformation and fracture mechanics, computer technologies and computer engineering. Modern computational technologies of risk analysis allow performing numerical statistical experiments based on mathematical models of complicated disasters. That results in a high validity of risk assessment results.

\section{References}

[1] Barlow R., Proshan F. Mathematical Theory of Reliability, Wiley: New York, 1965.

[2] Perrow Ch. Normal Accidents: Living with High-Risk Technologies, N.Y.: Basic Books, 1984.

[3] Bak P., Tang C., Wiesenfeld K. Self-organized criticality, Phys. Rev. A. V.38, N1, p.364-374. 1988.

[4] Arnold V. Catastrophe theory, M.: Nayka, 1990.

[5] Poston T., Stewart I. Catastrophe theory and its applications, M.: Mir, 1980.

[6] Malinetskiy G.G., Potapov A.B. Contemporary problems of the non-linear dynamics, M.: Editorial URSS, 2002.

[7] Bolotin V. The resource of machines and constructions. M.: Mashinostroenie, 1990.

[8] Makhutov N. Structural strength, resource and anthropogenic security. Part 2. Substantiation of the resource and security, Novosibirsk: Nauka, 2005.

[9] Lepikhin A., Moskvichev V., Cherniaev A. Risk analysis of the technical systems, Novosibirsk: Nauka, 2003. 